\title{
Stellar models: firm evidence, open questions and future developments
}

\author{
Santi Cassisi ${ }^{1}$ \\ ${ }^{1}$ INAF - Astronomical Observatory of Teramo, Via M. Maggini, 64100 Teramo, Italy \\ email: cassisi@oa-teramo.inaf.it
}

\begin{abstract}
During this last decade our knowledge of the evolutionary properties of stars has significantly improved. This result has been achieved thanks to our improved understanding of the physical behavior of stellar matter in the thermal regimes characteristic of the different stellar mass ranges and/or evolutionary stages.

This notwithstanding, the current generation of stellar models is still affected by several, not negligible, uncertainties related to our poor knowledge of some thermodynamical processes and nuclear reaction rates, as well as the efficiency of mixing processes. These drawbacks have to be properly taken into account when comparing theory with observations, to derive evolutionary properties of both resolved and unresolved stellar populations.

In this paper we review the major sources of uncertainty along the main evolutionary stages, and emphasize their impact on population synthesis techniques.
\end{abstract}

\section{Introduction}

As far as stellar model input physics is concerned, significant improvements have been achieved in the determination of the Equation of State (EOS) of the stellar matter, opacities, nuclear cross sections, neutrino emission rates. At the same time, stellar models computed with this updated physics have been extensively tested against the latest observational constraints.

The capability of the latest generation of stellar models to account for all the evolutionary phases observed in stellar clusters is undoubtedly an exciting achievement, that crowns with success the development of stellar evolutionary theories as pursued during the whole second half of the last century. Following this success, one is often tempted to use evolutionary results in an uncritical way, i.e., by taking these results at face value, without accounting for the associated uncertainties. However, theoretical uncertainties do exist, as it is clearly shown by the not negligible differences among the results obtained by different research groups.

A careful discussion of the uncertainties affecting stellar models for low-mass stars was early addressed by Chaboyer (1995), who investigated the reliability of theoretical models for H-burning stars presently evolving in galactic globular clusters (GGCs). This type of investigation has been extended to later evolutionary phases by Cassisi et al. (1998, 1999), Castellani \& Degl'Innocenti (1999), and Gallart et al. (2005). A discussion of the drawbacks of stellar models for low-mass stars and their impact on widely employed age, distance and chemical composition diagnostics has been also provided by Cassisi (2005 and references therein), whilst the same issues in case of intermediate-mass stellar models have been reviewed by Cassisi (2004). 
Stellar evolution models represent also a key ingredient for stellar population synthesis (SPS) tools, applied to the study of unresolves stellar populations. The accuracy and reliability of the adopted evolutionary framework affects therefore also our ability to derive physical properties of galaxies when employing SPS techniques. Until a few years ago, the stellar libraries adopted in SPS modelling were used with an uncritical approach, without taking care of their level of accuracy and completeness. Luckily enough, in recent times it is becoming increasingly clear that assessing the reliability of the adopted stellar models is a pivotal step to evaluate quantitatively the uncertainty of SPS results.

\section{On the impact of stellar models uncertainties on SPS predictions}

Stellar models can be affected by significant uncertainties that will affect SPS predictions. However, so far, stellar models have been usually emploted by the SPS community in an uncritical way. As a consequence, when applying SPS models to observations of both resolved and unresolved stellar populations, no one has considered the contribution to the systematic error that can affect, for instance, the derived ages and metallicities, coming from systematic uncertainties in the adopted model library.

The main reasons for this neglect are most probably due to the following: i) to check the effects of stellar model uncertainties on SPS predictions is an extremely time-consuming procedure - since one has to verify separately how the uncertainties in the various evolutionary stages affect the various SPS indicators (integrated colors, spectra and photometric indices); ii) it is sometime extremely difficult to obtain realistic estimates of the 'true' errors affecting the various stellar model predictions as evolutionary lifetimes, luminosity and effective temperature; iii) to test the impact of different, independent stellar model libraries is often problematic due to the difficulty of incorporating a stellar library in a SPS code.

This notwithstanding, there is now an ongoing effort in this direction (Gallart et al. 2005, Coelho et al. 2007, Lee et al. 2009a, 2009b, Cenarro et al. 2008, Conroy et al. 2009, Percival \& Salaris 2009, and references therein). For a detailed discussion, we refer the interested reader to the quoted papers. However, we wish to summarize here the main conclusions of these analyses. Some of the quoted investigations have shown that the various SPS diagnostics are affected differently and, sometimes, in the opposite sense, by systematic changes in the stellar model predictions, such as luminosity and/or effective temperature, and slight offsets between the metallicity scales of the adopted stellar model set and spectral library. This occurrence has a noteworthy implication for methods which fit simultaneously to several spectral indices for deriving ages and metallicities of unresolved stellar populations, since a failure to match several indices simultaneously could, spuriously, be interpreted for example as an indication of a non scaled-solar heavy elements distribution. It has been also proven that the inclusion of the Asymptotic Giant Branch (AGB) stage in SPS models is fundamental for understanding the physical properties of galaxies. However, a different treatment of this uncertain evolutionary stage alters the final results significantly as, for instance, the inferred galaxy masses (Bruzual 2007). Therefore, SPS models that do not account for the current uncertainties in AGB modelling are largely underestimating errors and may even be introducing systematic biases.

As for the possibility of testing the impact of independent stellar model databases in SPS tools, the situation has significantly improved in these last few years thanks to the availability of new, updated and complete sets of stellar models (Pietrinferni et al. 2004, 2006, Dotter et al. 2007, Bertelli et al. 2008 and references therein) that can be easily incorporated in a SPS code. Ideally, the SPS community should now make the effort of 
considering these different stellar model libraries in the SPS codes, in order to evaluate the effect of using independent model prescriptions on their SPS results.

The final point that has to be addressed is how to provide a realistic estimate of the uncertainties affecting the various stellar models predictions. Clearly, this information has to be provided by people working in the field of stellar evolution, by checking the impact of different physical inputs and/or physical assumptions on their own model computations, and comparing models provided by various authors.

\section{Stellar models: the state-of-the-art}

An in-depth discussion of the accuracy and reliability of contemporary stellar model predictions would be clearly beyond the scope of this paper. For such a reason, we wish to address only the major open problems affecting the model computations. In particular, we want to emphasize the uncertainties associated with the treatment of mass loss during the 'cool' evolutionary stages, i.e. the Red Giant Branch (RGB) and the AGB, and evolution of stars during the Thermal Pulses stage (TPAGB). However, we consider also useful to provide some indications about the level of reliability of model predictions concerning 'less problematic' evolutionary stages:

\subsection{The central H-burning stage}

In the last decade, the accuracy of central H-burning (main sequence) theoretical models has improved a lot. This occurrence is due to the availability of updated and accurate predictions concerning both the thermal and opacitive properties of matter in the relevant regime for both the interiors and atmospheres of low- and intermediate-mass stars. Some residual uncertainty is associated to (some) nuclear reaction rates. A large effort has been devoted to improve the measurements at energies as close as possible to the Gamow peak, i.e. the energies at which nuclear reactions occur in the stars. Thanks to this effort the nuclear processes involved in the $p-p$ chain have a small uncertainty. As a consequence, the uncertainty on the age - luminosity calibration is also negligible $(<2 \%)$. However, near the end of the main sequence, due to the paucity of $\mathrm{H}$, the energy supplied by the $\mathrm{H}-$ burning becomes insufficient and the star reacts contracting its core in order to produce the requested energy via gravitation. As a consequence, both central temperature and density increase and, when the temperature attains a value of $\sim 15 \times 10^{6} \mathrm{~K}$, the H-burning process is controlled by the $C N O$ cycle, whose efficiency is critically dependent on the ${ }^{14} N(p, \gamma){ }^{15} \mathrm{O}$ reaction rate, since this is the slowest reaction of the whole cycle.

Until few years ago, the rate for the ${ }^{14} N(p, \gamma){ }^{15} O$ reaction was uncertain, by a factor of 5 at least, because all available laboratory measurements were performed at energies well above the range of interest for astrophysical purposes (Angulo et al. 1999). The LUNA experiment (Formicola et al. 2003) has significantly improved the low energy measurements, obtaining an estimate which is about a factor of 2 lower than previous determinations. This lower rate for the ${ }^{14} N(p, \gamma){ }^{15} O$ reaction leads to a brighter and hotter Turn Off for a fixed age. The impact of this new rate on the age - luminosity relation (Imbriani et al. 2004) is the following: for a fixed TO brightness the new calibration predicts systematically older cluster ages, $\sim 0.9 \mathrm{Gyr}$ on average.

\subsection{The Red Giant Branch}

RGB stars are cool, reach high luminosities, their evolutionary timescales are relatively long, and therefore provide a major contribution to the integrated bolometric magnitude and to integrated colors and spectra at wavelengths larger than about $900 \mathrm{~nm}$ in old, unresolved stellar populations (e.g. Renzini \& Fusi-Pecci 1988; Worthey 1994). A correct 
theoretical prediction of the RGB spectral properties and colors is thus of paramount importance for interpreting observations of distant stellar clusters and galaxies using population synthesis methods, but also for determining the ages of resolved stellar systems by means of isochrone fitting techniques.

Both the RGB location and slope in the CMD are strongly sensitive to the metallicity, and for this reason, they are widely used as metallicity indicators.

The $I$-band brightness of the tip of the RGB (TRGB) provides a robust standard candle, largely independent of the stellar age and initial chemical composition, which allows to estimate distances out to about $10 \mathrm{Mpc}$ using HST observations. Due to the lingering uncertainties on the empirical determination of the TRGB brightness zero point, RGB models provide an independent calibration of this important standard candle (Salaris \& Cassisi 1997, 1998). Moreover, theoretical predictions about the structural properties of RGB stars at the Tip of the RGB play a fundamental role in determining the main evolutionary properties of their progeny: the core He-burning stars during the Horizontal Branch (HB) evolutionary phase. In particular, HB luminosities (like the TRGB ones) are mostly determined by the value of the electron degenerate He-core mass $\left(M_{\text {core }}^{H e}\right)$ at the end of the RGB evolution.

Predicted evolutionary timescales along the RGB phase play also a fundamental role in the determination of the initial He abundance of globular cluster stars through the $\mathrm{R}$ parameter (Salaris et al. 2004 and references therein).

A detailed analysis of the existing uncertainties in theoretical RGB models, and of the level of confidence in their predictions has been performed by Salaris et al. (2002). As far as the location and slope of RGB evolutionary tracks is concerned, model predictions are affected by: the EOS, the low-T opacity, the efficiency of superadiabatic convection, the choice about the model outer boundary conditions and the initial chemical abundances. It has been already emphasized that in the thermal regime appropriate for RGB stars, big improvements have been achieved concerning both the EOS and low-T opacity.

We think that it is worthwhile to discuss more in detail the issue related to the efficiency of the outer convection. As well known, the efficiency of superadiabatic convection parametrized by the mixing length parameter $\left(\alpha_{\mathrm{MLT}}\right)$ is usually calibrated by reproducing

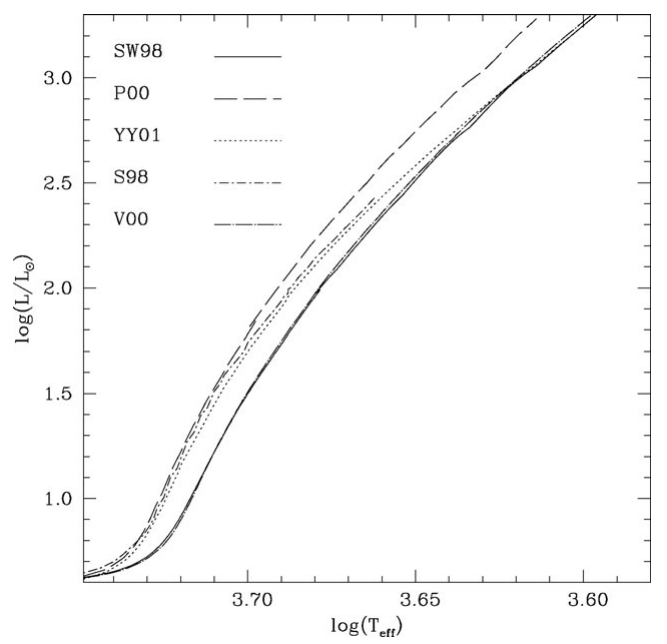

Figure 1. Comparison between the theoretical RGB tracks provided by various authors for the same assumptions about the age and chemical composition (see text for more details) (from Salaris et al. 2002). 
the solar $T_{\text {eff }}$, and this solar-calibrated value is then used for stellar models of different masses and along different evolutionary phases, including the RGB one. The adopted procedure guarantees that models always predict correctly the $T_{\text {eff }}$ of at least solar type stars. However, the RGB location is much more sensitive to the value of $\alpha_{\text {MLT }}$ than the main sequence. Therefore, it is important to verify that a solar $\alpha_{\mathrm{MLT}}$ is suitable also for RGB stars of various metallicities.

A source of concern about an a priori assumption of a solar $\alpha_{\mathrm{MLT}}$ for RGB computations comes from the fact that recent models from various authors, all using a suitably calibrated solar value of $\alpha_{\mathrm{MLT}}$, do not show the same RGB temperatures. This means that - for a fixed empirical RGB temperature scale - the calibration of $\alpha_{\mathrm{MLT}}$ based on RGB $T_{\text {eff }}$ estimates values would not provide always the solar value. Figure 1 displays several isochrones produced by different groups, all computed with the same initial chemical composition, same opacities, and the appropriate solar calibrated values of $\alpha_{\mathrm{MLT}}$ : Vandenberg et al. (2000, V00) and Salaris \& Weiss (1998, SW98) models are identical, the Padua ones (Girardi et al. 2000, P00) are systematically hotter by $\sim 200 \mathrm{~K}$, while the YY01 ones (Yi et al. 2001) have a different shape. This comparison shows clearly that if one set of solar calibrated RGBs can reproduce a set of empirical RGB temperatures, the others cannot, and therefore in some case a solar calibrated $\alpha_{\mathrm{MLT}}$ value may not be adequate. The reason for these discrepancies must be due to some difference in the input physics, like the EOS and/or the boundary conditions, which is not compensated by the solar re-calibration of $\alpha_{\mathrm{MLT}}$ (see also Vandenberg et al. 2008).

This occurrence clearly points out the fact that one cannot expect the same RGB $T_{\text {eff }}$ from solar calibrated models that do not employ exactly the same input physics. Therefore it is always necessary to compare RGB models with observations to ensure the proper calibration of $\alpha_{\mathrm{MLT}}$ for RGB stars. In the meantime, from the previous comparison, we can safely estimate that current uncertainties on the $T_{\text {eff }}$ scale of RGB models are of the order of $200-300 \mathrm{~K}$.

An other important prediction provided by RGB models is the number of stars in any given bin of the RGB luminosity function (LF - star counts as a function of brightness) which is determined by the local evolutionary rate. Therefore, the comparison between empirical and theoretical RGB LF represents a key test for the accuracy of the predicted RGB timescales (Renzini \& Fusi Pecci 1988). In addition, there are many more reasons why an investigation of RGB star counts is important, for instance: i) being the RGB stars among the brightest objects in a galaxy, their number has a strong influence on the integrated properties of the galactic stellar population; ii) the number ratio between RGB and stars along the AGB can be used to constrain the Star Formation History of a galaxy (Greggio 2000).

The investigation of the accuracy of theoretical RGB LFs has been performed by Zoccali \& Piotto (2000) by adopting a large database of GGC RGB LFs. The main outcome of their analysis was the evidence of, on average, a good agreement, in the whole explored metallicity range, between observations and the adopted theoretical predictions (but see also Sandquist \& Martel 2007).

An important quantity provided by models is the luminosity of the TRGB. The observational and evolutionary properties of RGB stars at the TRGB play a pivotal role in current stellar astrophysical research. As mentioned before, the reasons are manifold: i) the mass size of the He core at the He flash fixes not only the TRGB brightness but also the luminosity of the Horizontal Branch, ii) the TRGB brightness is one of the most important primary distance indicators.

As for the uncertainties affecting theoretical predictions about the TRGB brightness, it is clear that, being this quantity fixed by the He core mass, any uncertainty affecting the 
predictions of $M_{\text {core }}^{H e}$ immediately translates into an error on $\mathrm{M}_{\mathrm{bol}}^{\mathrm{TRGB}}$. An exhaustive analysis of the physical parameters that affect the estimate of $M_{\text {core }}^{H e}$ can be found in Salaris et al. (2002). Suffice to remember that the physical inputs that have the largest impact in the estimate of $M_{\text {core }}^{H e}$ are the efficiency of atomic diffusion and the conductive opacity. Unfortunately, no updates are available concerning a more realistic estimate of the real efficiency of diffusion in low-mass stars, apart from the Sun. On the contrary, concerning the conductive opacity, large improvements have been obtained recently (Potekhin 1999, Cassisi et al. 2007). This new set represents a significant improvement (both in the accuracy and in the range of validity) with respect to previous estimates.

We show in fig. 2 the comparison of the most recent results (Bertelli et al. 2008 - Padua, Pietrinferni et al. 2004 - BaSTI, Vandenberg et al. 2000 - Victoria, Dotter et al. 2007 Dartmouth, Yi et al. 2001 - Yonsei-Yale) concerning the TRGB bolometric magnitude and $M_{\text {core }}^{H e}$ at the He-flash; the displayed quantities refer to a $0.8 M_{\odot}$ model and various initial metallicities. When excluding the Padua models, there exists a fair agreement among the various predictions about $M_{c o r e}^{H e}$ : at fixed metallicity the spread among the various sets of models is at the level of $0.003 M_{\odot}$. For the Padua models, we show the results corresponding to the two different initial He contents adopted by the authors: we have no clear explanation for the fact that the Padua models predict the lowest values for $M_{\text {core }}^{\mathrm{He}}$, as well as for the presence of an 'erratic' behavior of the values corresponding to the different He abundances: for a fixed total mass and metallicity, the $M_{\text {core }}^{H e}$ value is expected to be a monotonic function of the initial He abundance. Concerning the trend of $\mathrm{M}_{\mathrm{bol}}^{\mathrm{TRGB}}$, all model predictions at a given metallicity are in agreement within $\sim 0.15$ mag, with the exception of the Padua models that appear to be brighter, at odds with the fact that they predict the lowest $M_{\text {core }}^{H e}$ values. In case of the Yonsei-Yale models, the result is also surprising since the fainter TRGB luminosity cannot be explained by much smaller $M_{\text {core }}^{\mathrm{He}}$ values, because this quantity is very similar to, for instance, the results given by Vandenberg et al. (2000). When neglecting the Padua and Yonsei-Yale models,

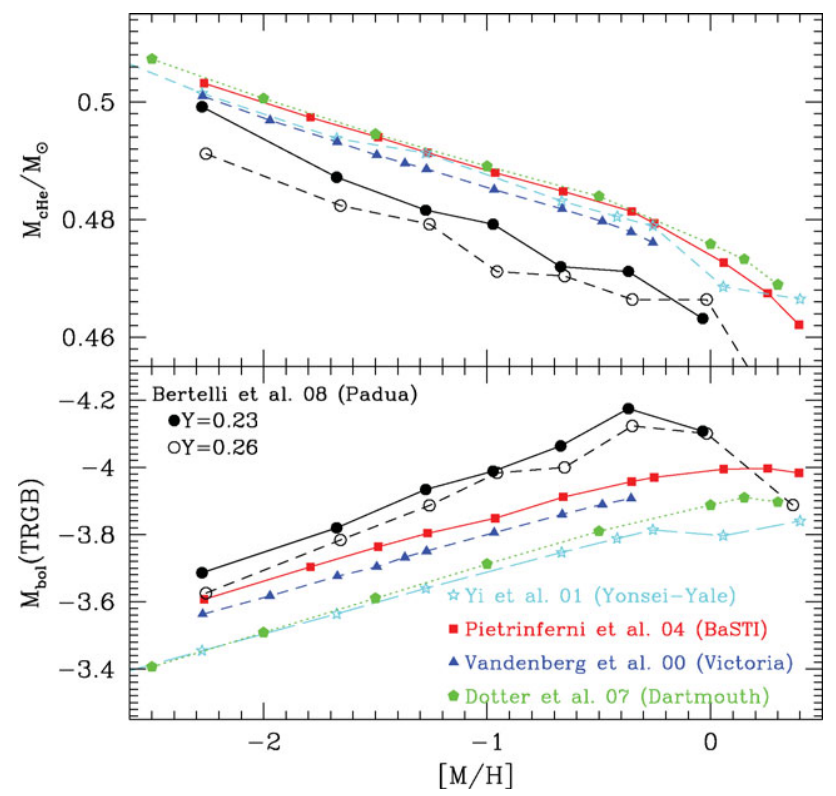

Figure 2. The trends of $M_{\text {core }}^{H e}$ and $\mathrm{M}_{\mathrm{bol}}^{\mathrm{TRGB}}$ as a function of the metallicity as provided by the most recent stellar model libraries (see text for more details). 
the $\sim 0.1$ mag spread among the different TRGB brightness estimates can be interpreted in terms of differences in the adopted physical inputs such as for instance the electron conduction opacities.

Due to its relevance as standard candle, it is worthwhile showing a comparison between theoretical predictions about the I-Cousins magnitude of the TRGB and empirical calibrations. This comparison is displayed in fig. 3, where we show also the recent empirical calibration provided by Bellazzini et al. (2001) based on the well populated GGC $\omega$ Cen. In this plot, we have shown different calibrations of $M_{I}^{T R G B}$ as a function of the metallicity based on our own stellar models. These calibration are about 0.20 mag brighter than the most recent, empirical ones. However, it is also important to note the new calibration by Cassisi et al. (2007) - based on the new conductive opacity - is in better agreement with the empirical evidence.

\section{Stellar models: the open problems}

\subsection{The mass-loss efficiency}

One of the thorniest problems in current stellar evolution theory is that related to the efficiency of mass loss (ML) during both the RGB and AGB stage. In fact, the efficiency of ML during the RGB strongly controls the $T_{\text {eff }}$ - and hence the color - of the star along the HB stage, while during the AGB by reducing the envelope mass, it truncates the AGB evolution - and hence the contribution of the star to the infrared flux of the global stellar population.

The astrophysical impact of ML in both Pop. I and II giants is huge and affects also the interpretation of the UV excess in ellipticals, or the interaction between the cool intracluster medium and hot halo gas. There is so much indirect but quantitative evidence for ML during the giant branches evolution, namely the HB morphology and the $2^{\text {nd }}$ parameter problem, the pulsational properties of RR Lyrae, the absence of AGB stars brighter than the RGB tip and the masses of White Dwarfs in GCs (see Catelan 2009 and

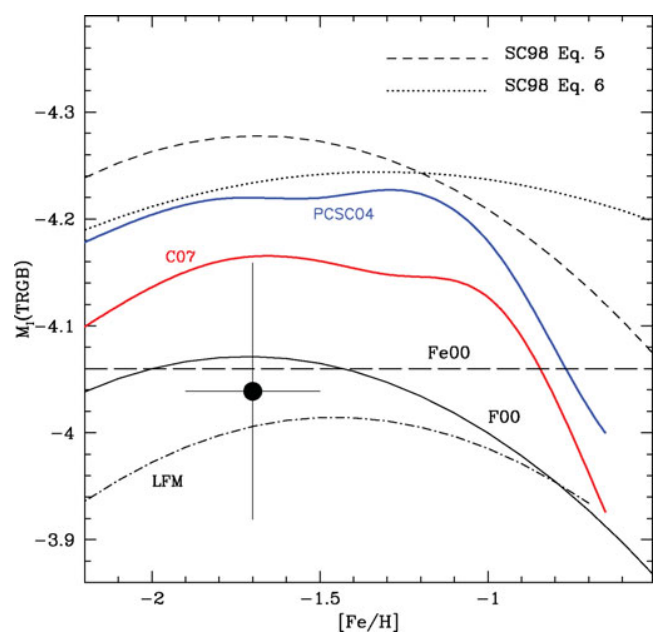

Figure 3. A comparison among theoretical calibrations of the I-Cousins magnitude of the TRGB as provided by Pietrinferni et al. (2004, PCSC04) and Salaris \& Cassisi (1998, SC98, see their equations 5 and 6), and (semi-)empirical ones as given by Lee et al. (1993, LFM), Ferrarese et al. (2000, Fe00) and Ferraro et al. (2000, F00). The new theoretical calibration by Cassisi et al. $(2007, \mathrm{C} 07)$ is also shown. The full circle with the error bars corresponds to the empirical calibration provided by Bellazzini et al. (2001). 
references therein). However, despite its importance, complete empirical determinations as well as a comprehensive physical description of the involved processes are still lacking. So far, there is a lack of any empirical law directly calibrated on Population II giants. Indeed, only a few, sparse estimates of ML for giants along the brightest portion of the RGB and AGB exist. From a theoretical point of view, our knowledge of the ML timescales, driving mechanisms, dependence on stellar parameters and metallicity is also very poor. The consequence is that there is little theoretical or observational guidance on how to incorporate ML into stellar model computations.

Without a better recipe, models of stellar evolution incorporate ML by using analytical formulae calibrated on Population I bright giants, the first and most used being the Reimers (1975) formula, extrapolated towards lower luminosity by also introducing a free parameter $\eta$ (typically 0.3 ), to account for a somewhat less efficient ML along the RGB. A few other formulae, which are variants of the Reimers one, have been proposed in the subsequent years (see Catelan 2009 for a detailed discussion on this issue) but there is no a priori reason for choosing among the different alternatives. In these last few years, at least on the observational side, the situation is improving thanks to the availability of the observational facilities associated to, for instance, the SPITZER telescope. As a consequence, there is growing amount of empirical data concerning ML estimates for Pop. II red giants (see Origlia et al. 2007 and references therein).

The preliminary empirical ML law that has been obtained (Origlia et al. 2007) appears significantly different (flatter) than the Reimers formula, that appears to be ruled out by current empirical estimates at the $3 \sigma$ level. In addition, it seems that the ML phenomenon is not a continuous process along the RGB but an episodic phenomenon, and it does not appear to be strongly correlated with the cluster metallicity.

The situation is still more controversial and complicated in the case of AGB stars, due to the link existing between the ML efficiency (and the physical processes that causes the ML) and the evolutionary, structural and pulsational properties of the evolving star (see van Loon 2008 for a detailed review on this issue).

We expect that, thanks to the availability of new observational facilities and the huge theoretical and observational effort that is devoted to this subject, significant improvements could be obtained in the next decade. This occurrence would contribute, not only to obtain more accurate stellar models, but also in reducing the degrees of freedom in the SPS modeling.

\subsection{The AGB stage modeling}

The computation of AGB stellar models is one of the most complicated task for the stellar evolution community. The reasons for this has to be found in the realization that the evolutionary properties of these stars are hugely dependent on the complex link existing among mixing processes (the 3DU), ML efficiency, nucleosynthesis and envelope opacity stratification. The evolutionary results that can be obtained, strongly depend on the assumptions about the efficiency of these various processes - and their treatment in the numerical codes - adopted in the model computations.

Concerning the 3DU, in spite of its fundamental relevance in determining the chemical enrichment of TPAGB star envelopes, its treatment in stellar evolutionary code is quite uncertain. This is due to the fact that we are not yet able to properly describe convection inside the stars, and in particular, in case of mixing occurring in a region with a strong composition/opacity discontinuity. Many different - arbitrary - methods can be envisaged to treat the occurrence of the 3DU, but each one of these approaches has no robust physical ground. This has the important implication that in all fully evolutionary AGB models, the efficiency of the 3DU is managed by using one (or more) free parameter(s). 
An important physical implication of the occurrence of the 3DU is the huge change in the envelope $\mathrm{C} / \mathrm{O}$ ratio that is caused by this process (Ventura \& Marigo 2009). The change in the $\mathrm{C} / \mathrm{O}$ ratio when it approaches (and overcomes) unity has huge effects on the opacitive properties of the stellar envelope (Marigo 2002). As a consequence, the $\mathrm{C} / \mathrm{O}$ ratio drives sharp discontinuities in many observational properties of AGB stars: $T_{\text {eff }}$, colors, spectra, mass loss efficiency, etc. After many years during which only approximate evaluations for the C-rich mixture opacity were available, the situation is now largely improving and new opacity tables for AGB envelopes can be now incorporated in evolutionary codes (Cristallo et al. 2008, Weiss \& Ferguson 2009). These new opacities, although in good qualitative agreement with the previous estimates, show significant, quantitative differences. This occurrence has the effect that the $T_{\text {eff }}$ scale for AGB models is expected to be significantly affected, with consequences on SPS modeling that are still to be fully exploited.

Before concluding, we wish to comment briefly on synthetic AGB modeling (see, e.g., Marigo et al. 2008). These synthetic simulations are based on analytical, functional relations providing the link among the main structural - such as the He core mass - evolutionary properties - as $T_{\text {eff }}$ and luminosity - and chemical properties - mainly the metallicity and the $\mathrm{C} / \mathrm{O}$ ratio -, obtained from the numerical integration of the stellar structure and envelope. The (recent) literature - see also the proceedings of this meeting - contains several claims about the fact that the new synthetic AGB computations are significantly better and more accurate than previous ones, allowing a better (or "perfect'!) match to various empirical constraints. In our belief, these claims are somewhat misleading. Even though large improvements have been achieved in the field of AGB star modelling (see above), the same problems affecting the full evolutionary models affect also synthetic AGB modeling. As a consequence, in these synthetic simulations there is a number of free parameters that have to be tuned 'by hand' in order to reproduce some observational constraints such as for instance the Magellanic Clouds C-stars luminosity functions. Synthetic AGB models constitute a much faster way to produce approximate models with these parameters tuned to match some observations, but it is not clear what their predictive power is, outside the parameter space covered by the calibrating AGB populations. We think this is an important warning that should be kept in mind by the SPS community, in order to avoid overestimating the reliability of the SPS tools that incorporate the new generations of synthetic AGB models.

It will not be possible to have a realistic and physically well grounded treatment of the AGB stage, until we will account for the various physical processes at work in AGB stars starting from a robust theory, free of tunable parameters.

\section{Acknowledgements}

We warmly acknowledge M. Salaris for his pertinent suggestions and for reading an early draft of this manuscript. We are also grateful to him for many enlightening discussions. We thank also A. Pietrinferni for his continuous help. We warmly thank the LOC and the SOC for organizing this stimulating meeting.

\section{References}

Angulo, C. et al. 1999, Nuclear Physics A, 656, 3

Bellazzini, M., Ferraro, F. R., \& Pancino, E. 2001, ApJ, 556, 635

Bertelli, G., Girardi, L., Marigo, P., \& Nasi, E. 2008, A\& $A, 484,815$

Bruzual, G. 2007, in IAU Symp. 241 'Stellar Populations as Building Blocks of Galaxies', ed. A. Vazdekis \& R. F. Peletier (Cambridge: Cambridge Univ. Press), 125 
Cassisi, S. 2004, IAU Colloquium 193, 'Variable Stars in the Local Group', D. W. Kurtz and K.

R. Pollard, ASP Conference Proceedings (San Francisco), Vol. 310, p. 489

Cassisi, S. 2005, in , D. Valls-Gabaud \& M. Chávez (eds), Proc. of the meeting 'Resolved Stellar Populations', in press (astro-ph/0506161)

Cassisi, S., Castellani, V., Degl'Innocenti, S., Salaris, M., \& Weiss, A. 1999, A\& ASS, 134, 103

Cassisi, S., Castellani, V., Degl'Innocenti, S., \& Weiss, A. 1998, A $\& A S S, 129,267$

Cassisi, S., Potekhin, A., Pietrinferni, A., Catelan, M., \& Salaris, M. 2007, ApJ, 661, 1094

Castellani, V. \& Degl'Innocenti, S. 1999, A\&A 344,97

Catelan, M. 2009, Ap\&SSS, 320, 261

Cenarro, A. J. et al. 2008, ApJ, 698, L29

Chaboyer, B. 1995, ApJ, 444, L9

Coelho, P. et al. 2007, MNRAS, 382, 498

Conroy, C., Gunn, J. E., \& White, M. 2009, ApJ, 699, 486

Cristallo, S., Straniero, O., Lederer, M. T., \& Aringer, B. 2007, ApJ, 667, 489

Dotter, A. et al. 2007, AJ, 134, 376

Ferrarese, L. et al. 2000, ApJS, 128, 431

Ferraro, F. R., Montegriffo, P., Origlia, L., \& Fusi Pecci, F. 2000, AJ, 119, 1282

Formicola, A. et al. 2003, Nuclear Instruments and Methods in Physics Research Section A, 507, 609

Gallart, C., Zoccali, M. \& Aparicio, A. 2005, ARAA, 43, 387

Girardi, L., Bressan, A., Bertelli, G., \& Chiosi, C. 2000, A\& ASS, 141, 371

Greggio, L. 2000, Proceeding of the meeting 'Observed HR diagrams and Stellar Evolution', ASP Conf. Proceeding, T. Lejeune and J. Fernandes eds., vol. 274, p. 444

Imbriani, G. et al. 2004, A\& A, 420, 625

Lee, M. G., Freedman, W., \& Madore, B. F. 1993, ApJ, 417, 553

Lee, H.-c, et al. 2009a, ApJ, 694, 902

Lee, H.-c, Worthey, G., \& Blakeslee, J. P. 2009b, ApJ, in press, (astro-ph/0902.1177)

Marigo, P. 2002, A\&A, 387, 507

Marigo, P. et al. 2008, A\&A, 482, 883

Origlia, L. et al. 2007, ApJ, 667, 85

Percival, S. M. \& Salaris, M. 2009, ApJ, 703, 1123

Pietrinferni, A., Cassisi, S., Salaris, M., \& Castelli, F. 2004, ApJ, 612, 168

Pietrinferni, A., Cassisi, S., Salaris, M., \& Castelli, F. 2006, ApJ, 642, 797

Potekhin, A. Y. 1999, A\&A, 351, 787

Reimers, D. 1975, Mem. Soc. R. Sci. Liège 6 Ser., 8, 369

Renzini, A. \& Fusi Pecci, F. 1988, ARAA, 26, 199

Salaris, M. \& Cassisi, S. 1997, MNRAS, 289, 406

Salaris, M. \& Cassisi, S. 1998, MNRAS, 298, 166

Salaris, M., Cassisi, S., \& Weiss, A. 2002, PASP, 114, 375

Salaris, M., Riello, M., Cassisi, S., \& Piotto, G. 2004, A\&SA, 420, 911

Salaris, M. \& Weiss, A. 1998, A\& A, 335, 943

Sandquist, E. L. \& Martel, A. R. 2007, ApJ, 654, 65

VandenBerg, D. A. et al. 2000, ApJ, 532, 430

VandenBerg, D. A. Edvardsson, B., Eriksson, K., \& Gustafsson, B. 2008, ApJ, 675, 746

van Loon, J.Th. 2008, Mem. Soc. Astr. It., 79, 412

Ventura, P. \& Marigo, P. 2009, MNRAS, in press, (astro-ph/0907.3204)

Weiss, A. \& Ferguson, J. 2009, A\&SA, in press, (astro-ph/0903.2155)

Worthey, G. 1994, ApJS, 95, 107

Yi, S. et al. 2001, ApJS, 136, 417

Zoccali, M. \& Piotto, G. 2000, A\&A, 358, 943 